\title{
Genitalia Preserving Robotic Radical Cystectomy with Intracorporeal Studer Pouch Formation in the Female: Experience in 5 Cases
}

\author{
Ersin Koseoglu (D) \\ Mert Kilic ${ }^{2}$ \\ Arif Ozkan' \\ Kayhan Tarim (iD) \\ Abdullan Erdem Canda (D) \\ Mevlana Derya Balbay (D) 1,2 \\ 'Koç University, School of Medicine, \\ Department of Urology, Istanbul, Turkey; \\ ${ }^{2}$ VKF American Hospital, Department of \\ Urology, Istanbul, Turkey
}

\begin{abstract}
We present operative, postoperative, oncologic and functional outcomes of genital tract sparing robot-assisted laparoscopic radical cystectomy, bilateral extended pelvic lymph node (LN) dissection and intracorporeal Studer pouch construction on five female patients with bladder cancer. One of the cases had concomitant nephroureterectomy performed due to a non-functioning kidney. Median patient age, mean operation time, median estimated blood loss and mean duration of hospital stay were 59 (39-78), $462 \pm 25,400$ (50-970), $8.8 \pm 2.5$, respectively. Pathologic stages were pTis $(n=1), p T 1(n=1), p T 3(n=1)$, pT4a $(n=1)$ and pT4b $(n=1)$. Mean LN yield was $32.4 \pm 8.9$. Positive surgical margins were detected in 2 patients with pT4 diseases. Only minor complications developed that were resolved with medical treatment. Two patients had no, 1 patient had mild, and 2 patients had moderate daytime incontinence. One patient had good, 3 patients had fair, and 1 had poor night-time incontinence. This complex robotic surgery can be performed safely with acceptable short-term surgical, oncological and functional outcomes.
\end{abstract}

Keywords: female robotic cystectomy, genital tract preservation, intracorporeal Studer pouch, paravaginal nerve sparing, bladder cancer

\section{Introduction}

Gynecologic-tract sparing radical cystectomy (RC) in female patients with bladder cancer has attracted attention. Although an increasing number of publications exists in the literature related to robot-assisted laparoscopic radical cystectomy (RARC) in females, to date, very few authors have described gynecologic-tract sparing RARC. ${ }^{1-5}$ Herein, we report surgical, oncologic and functional outcomes of five cases where we performed gynecologic-tract sparing RARC with intracorporeal Studer pouch reconstruction.

\section{Materials and Methods}

This study was conducted in accordance with the declaration of Helsinki. Informed consent to participate in the study prior to the study commencement was obtained from the patients. Patients also gave consent to have their data published. Following internal review board approval of Koç University, five female patients with muscle invasive urothelial carcinoma in the bladder were included. The selection criteria for the technique were patients' choice and tumors located except bladder neck or tumors with no suspicion of uterine infiltration. Radiologic work-up revealed no systemic
Correspondence: Ersin Koseoglu Koç University, School of Medicine, Department of Urology, Zeytinburnu, Istanbul, 34010, Turkey

Tel +905306930442

Email ekoseoglu@kuh.ku.edu.tr 
metastasis but a non-functioning kidney in one patient. Gynecologic-tract sparing RARC with bilateral extended pelvic lymph node (LN) dissection and intracorporeal Studer pouch reconstruction was performed in all patients (Figure 1). Additionally, a concomitant right robotic nephroureterectomy was performed in one patient. In the present case series, all patients underwent genitalia preserving RARC preserving the uterus, fallopian tubes, ovaries and vagina.

\section{Results}

Patients' characteristics, operative-postoperative parameters, pathologic parameters, complications and functional outcomes are presented in Table 1. Only minor complications developed that were resolved with medical treatment. Post-renal kidney failure developed in patient-3 during postoperative 31-90 days with grade-II hydronephrosis during ultrasound decreased urine output and increased serum creatinine. Bilateral double-J stents were inserted via flexible ureterorenoscopy. During follow-up, urine output increased and serum creatinine dropped down to normal limits. Double-J stents were removed after 3 months (Table 1). None of our patients needed clean intermittent catheterization during follow-up.

\section{Discussion}

In the literature, a limited number of publications exist related to female cystectomy with preservation of the internal genital organs. Salem et al reported the pathological findings of gynecologic organs obtained in female RC $(n=250)$ performed for urothelial carcinoma. ${ }^{3}$ Malignancy was identified in 1 uterus and 11 vagina specimens, including invasive urothelial carcinoma, all of which were clearly identified intraoperatively. They concluded that preservation of internal genitalia should be considered in selected cases after careful preoperative assessment. ${ }^{6}$ In a larger study from Egypt, pathological findings of gynecologic organs obtained at female RC $(n=609)$ were reported and gynecologic organ involvement was detected in $2.6 \%$ of the cases. No primary genital cancers were identified. Gynecologic organ involvement was significantly more frequent in high-grade tumors and in transitional cell cancer type. Although statistically not significant, posterior wall tumors were more frequently associated with genital involvement compared to other sites. They also concluded that routine removal of uninvolved gynecologic organs during $\mathrm{RC}$ in women was not mandatory. ${ }^{7}$ Therefore, preservation of the internal genital
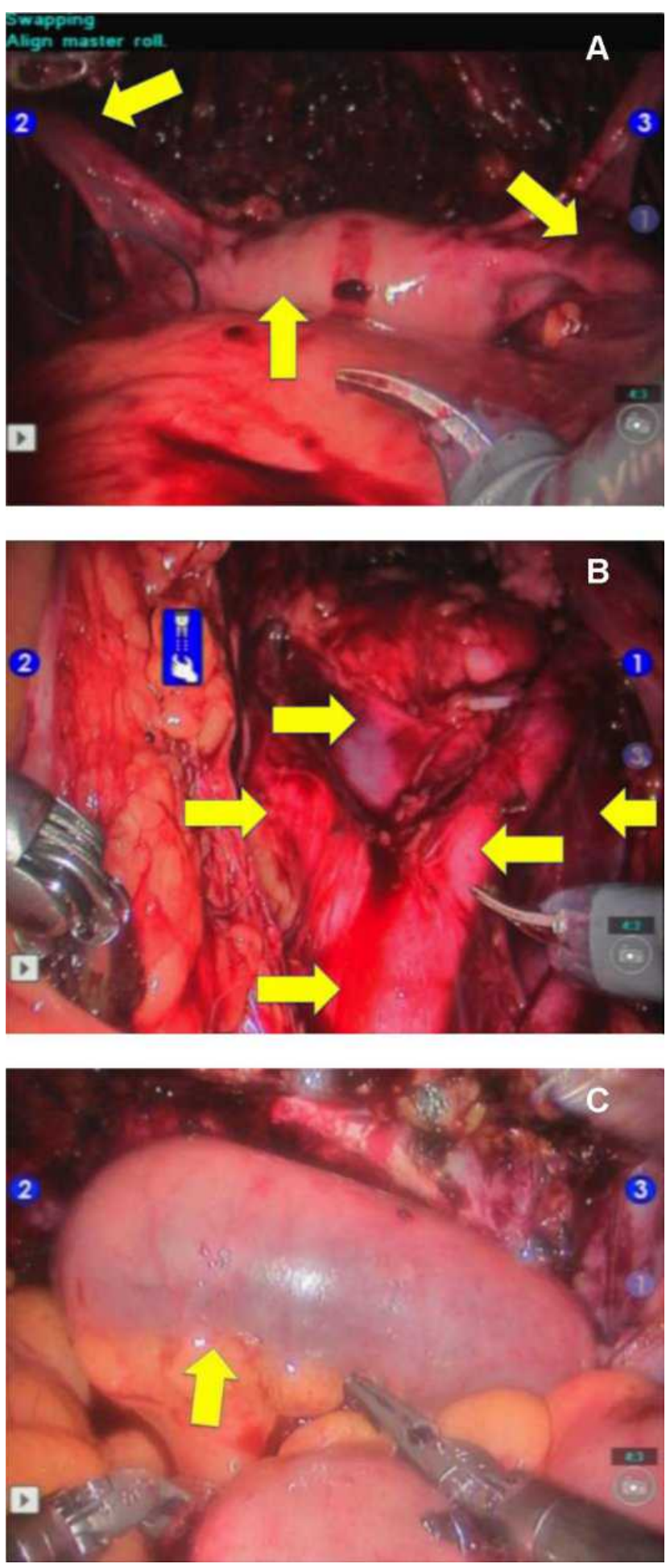

Figure I Steps of the operation. (A) Completed robot-assisted laparoscopic radical cystectomy with preservation of uterus and ovaries (arrows). (B) Appearance of extended pelvic lymph node dissection. Arrows: abdominal aorta and associated major vasculature. (C) Appearance of completed intracorporeal Studer pouch distended with sterile saline solution (arrow).

organs without any clinical suspicion of involvement during female RC seems to be safe. Additionally, Bai et al published a retrospective propensity score-matched study 
Table I Patient Characteristics Including Demographics, Operative-Postoperative Parameters, Pathologic Parameters, Complications and Functional Outcomes

\begin{tabular}{|c|c|c|c|c|c|}
\hline & Case I & Case 2 & Case 3 & Case 4 & Case 5 \\
\hline Patient age: (years) & 57 & 59 & 63 & 78 & 39 \\
\hline BMI: $\left(\mathrm{kg} / \mathrm{m}^{2}\right)$ & 40 & 26 & 35 & 24 & 24 \\
\hline ASA Score: & 3 & 2 & 2 & 3 & I \\
\hline $\begin{array}{l}\text { Previous } \\
\text { abdominal } \\
\text { surgery history: }\end{array}$ & None & $\begin{array}{l}\text { Appendectomy, } \\
\text { Cesarean section }\end{array}$ & $\begin{array}{c}\text { Laparoscopic } \\
\text { cholecystectomy }\end{array}$ & Appendectomy & Abdominoplasty \\
\hline $\begin{array}{l}\text { Co-morbid } \\
\text { diseases: }\end{array}$ & Hypertension & None & Hypertension & Hypertension & None \\
\hline Smoking history: & $\begin{array}{l}\text { I package/day } \\
\text { (40 years) }\end{array}$ & None & None & $\begin{array}{l}\text { I package/day } \\
\text { (20 years) }\end{array}$ & None \\
\hline $\begin{array}{l}\text { Presence of any } \\
\text { type of } \\
\text { preoperative } \\
\text { urinary } \\
\text { incontinence: }\end{array}$ & None & None & None & Urgency incontinence & None \\
\hline $\begin{array}{l}\text { Premenopausal/ } \\
\text { Postmenopausal } \\
\text { state: }\end{array}$ & Postmenopausal & Postmenopausal & Postmenopausal & Postmenopausal & Premenopausal \\
\hline $\begin{array}{l}\text { Bilateral NVB- } \\
\text { sparing: }\end{array}$ & Yes & Yes & Yes & Yes & Yes \\
\hline $\begin{array}{l}\text { Anomalies } \\
\text { detected during } \\
\text { surgery: }\end{array}$ & None & None & None & $\begin{array}{c}\text { Severe intra- } \\
\text { peritoneal adhesions }\end{array}$ & $\begin{array}{l}\text { Tumor infiltration } \\
\text { into pelvic bone }\end{array}$ \\
\hline $\begin{array}{l}\text { Estimated blood } \\
\text { loss: }(\mathrm{mL})\end{array}$ & 800 & 150 & 400 & 950 & 50 \\
\hline $\begin{array}{l}\text { Bilateral } \\
\text { extended lymph } \\
\text { node dissection: }\end{array}$ & Yes & Yes & Yes & Yes & Yes \\
\hline $\begin{array}{l}\text { Lymph node yield: } \\
\mathrm{n}\end{array}$ & 39 & 30 & 30 & 43 & 20 \\
\hline Urinary diversion: & Intracorporeal & Intracorporeal & Intracorporeal & Intracorporeal & Intracorporeal \\
\hline $\begin{array}{l}\text { Urinary diversion } \\
\text { type: }\end{array}$ & Studer & Studer & Studer & Studer & Studer \\
\hline $\begin{array}{l}\text { Concomitant } \\
\text { robotic surgery: }\end{array}$ & $\begin{array}{c}\text { Right radical } \\
\text { nephroureterectomy }\end{array}$ & None & None & None & None \\
\hline $\begin{array}{l}\text { Operative time: } \\
(\min )\end{array}$ & $\begin{array}{l}440 \\
+ \\
\text { I } 30 \text { (nephroureterectomy } \\
\text { time including patient re- } \\
\text { positioning and robot de- } \\
\text { docking and re-docking) }\end{array}$ & 370 & 480 & 540 & 480 \\
\hline
\end{tabular}

(Continued) 
Table I (Continued).

\begin{tabular}{|c|c|c|c|c|c|}
\hline & Case I & Case 2 & Case 3 & Case 4 & Case 5 \\
\hline Overall (hours): & 9.5 & 6.2 & 8 & 9 & 8 \\
\hline Radical cystectomy: & $150 \mathrm{~min}$ & $110 \mathrm{~min}$ & $150 \mathrm{~min}$ & 170 & 190 \\
\hline $\begin{array}{l}\text { Bilateral extended } \\
\text { lymph node } \\
\text { dissection: }\end{array}$ & $110 \mathrm{~min}$ & $90 \mathrm{~min}$ & $130 \mathrm{~min}$ & 210 & 120 \\
\hline $\begin{array}{l}\text { Intracorporeal } \\
\text { Studer pouch } \\
\text { reconstruction: }\end{array}$ & $180 \mathrm{~min}$ & $170 \mathrm{~min}$ & $200 \mathrm{~min}$ & 160 & 170 \\
\hline $\begin{array}{l}\text { Pathologic stage } \\
\text { (pT): }\end{array}$ & pT3 & pTis & PTI & $\mathrm{pT} 4 \mathrm{a}$ & pT4b \\
\hline $\begin{array}{l}\text { Number of } \\
\text { metastatic lymph } \\
\text { nodes: } n\end{array}$ & 5 & 0 & 0 & 2 & 0 \\
\hline Surgical margins: & Negative & Negative & Negative & Positive & Positive \\
\hline $\begin{array}{l}\text { Time to liquid } \\
\text { diet: (days) }\end{array}$ & 1 & 2 & 2 & 3 & 2 \\
\hline $\begin{array}{l}\text { Time to regular } \\
\text { diet: (days) }\end{array}$ & 6 & 3 & 3 & 7 & 3 \\
\hline $\begin{array}{l}\text { Time to } \\
\text { ambulation: (days) }\end{array}$ & I & 3 & 2 & 3 & 1 \\
\hline $\begin{array}{l}\text { Abdominal drain } \\
\text { removal time: } \\
\text { (days) }\end{array}$ & 6 & 7 & 7 & 8 & 8 \\
\hline $\begin{array}{l}\text { Length of hospital } \\
\text { stay: (days) }\end{array}$ & 6 & 8 & 8 & 13 & 9 \\
\hline $\begin{array}{l}\text { Follow-up: } \\
\text { (months) }\end{array}$ & 24 & 10 & 7 & 26 & 7 \\
\hline \multicolumn{6}{|l|}{$\begin{array}{l}\text { Complications } \\
\text { due to modified } \\
\text { Clavien } \\
\text { Classification: }\end{array}$} \\
\hline \multicolumn{6}{|l|}{$\begin{array}{l}\text { 0-30 day (Peri- } \\
\text { operative period) }\end{array}$} \\
\hline $\begin{array}{l}\text { Minor complication } \\
\text { (Grade I and 2): n }\end{array}$ & I: UTI & $\begin{array}{c}\text { 2: Abdominal pain } \\
\text { and UTI }\end{array}$ & None & $\begin{array}{c}\text { Abdominal pain and } \\
\text { UTI }\end{array}$ & Abdominal pain \\
\hline $\begin{array}{l}\text { Major complication } \\
\text { (Grade 3-5): n }\end{array}$ & None & None & None & None & None \\
\hline \multicolumn{6}{|l|}{$31-90$ day period } \\
\hline $\begin{array}{l}\text { Minor complication } \\
\text { (Grade I and 2): n }\end{array}$ & None & None & None & None & None \\
\hline
\end{tabular}

(Continued) 
Table I (Continued).

\begin{tabular}{|c|c|c|c|c|c|}
\hline & Case I & Case 2 & Case 3 & Case 4 & Case 5 \\
\hline $\begin{array}{l}\text { Major complication } \\
\text { (Grade } 3-5): n\end{array}$ & None & None & $\begin{array}{l}\text { Post-renal kidney } \\
\text { failure }\end{array}$ & None & None \\
\hline \multicolumn{6}{|l|}{$\begin{array}{l}\text { Postoperative } \\
\text { readmission rates }\end{array}$} \\
\hline \multicolumn{6}{|l|}{$\begin{array}{l}\text { 0-30 day (Peri- } \\
\text { operative period) }\end{array}$} \\
\hline $\begin{array}{l}\text { Due to minor } \\
\text { complications: } \mathrm{n}\end{array}$ & None & None & None & None & None \\
\hline $\begin{array}{l}\text { Due to major } \\
\text { complications: } \mathrm{n}\end{array}$ & None & None & None & None & None \\
\hline \multicolumn{6}{|l|}{$31-90$ day period } \\
\hline $\begin{array}{l}\text { Due to minor } \\
\text { complications: } n\end{array}$ & None & None & None & None & None \\
\hline $\begin{array}{l}\text { Due to major } \\
\text { complications: } \mathrm{n}\end{array}$ & None & None & $\begin{array}{c}\text { Bilateral double } \\
\text { J stents were inserted }\end{array}$ & None & None \\
\hline \multicolumn{6}{|l|}{$\begin{array}{l}\text { Postoperative } \\
\text { urinary } \\
\text { continence } \\
\text { evaluation: }\end{array}$} \\
\hline $\begin{array}{l}\text { Day-time } \\
\text { incontinence: } \\
\text { (None/Mild/ } \\
\text { Moderate/Severe) }\end{array}$ & $\begin{array}{c}\text { Moderate } \\
\text { (urge incontinence) }\end{array}$ & $\begin{array}{c}\text { Mild } \\
\text { (urge } \\
\text { incontinence) }\end{array}$ & None & $\begin{array}{c}\text { Moderate } \\
\text { (urge incontinence) }\end{array}$ & None \\
\hline $\begin{array}{l}\text { Night-time } \\
\text { incontinence: } \\
\text { (Good/Fair/Poor) }\end{array}$ & Fair & Fair & Fair & Poor & Good \\
\hline $\begin{array}{l}\text { Current sexual } \\
\text { function: }\end{array}$ & Sexually inactive & Sexually inactive & Sexually inactive & Sexually inactive & Sexually inactive \\
\hline
\end{tabular}

Notes: Complications were graded according to modified-Clavien system. Day-time urinary incontinence was measured as none (0-I security pad/day), mild (I-2 pads/day), moderate ( 3 pads/day) and severe (>3 pads/day) described by Lantz et al. Night-time urinary incontinence was measured as good (dry with no protection), fair (dry with one awakening) and poor (wet, leakage and incontinence during sleep) described by Kulkarni et al. ${ }^{4}$

Abbreviations: BMI, body mass index; ASA, American Society of Anesthesiologists; NVB, neurovascular bundle; UTI, urinary tract infection.

including 135 females who underwent RC. Among 51 reproductive organ sparing RC; operative time was shorter, bowel recovery was quicker, the incidence of complications and estimated blood loss was lower than only RC group ( $\mathrm{n}=84){ }^{8}$

Roshdy et al published their 7 years of genitalia sparing open cystectomy experience in 24 female patients. Pathological examination revealed only one locally advanced disease which could not detected by preoperative imaging studies in their study. In their 48 months of follow-up period, the overall survival rate was $91.6 \%$. Day time and night urinary continence rates were $95.4 \%$ and $90.2 \%$, respectively. ${ }^{3}$ Ali-El-Dein et al reported the outcomes of 15 female cases who underwent genitalia sparing open $\mathrm{RC}$ with orthotopic neobladder reconstruction. They included patients with clinical stage $\mathrm{T} 2 \mathrm{bN} 0 \mathrm{M} 0$ or less disease, unifocal tumors away from the trigone, sexually active young women and without any clinical suspicion of the involvement of internal genitalia by tumor. During a mean follow-up of $70 \pm 21$ months, no recurrence was detected in the retained genital organs. Among women eligible for functional evaluation, daytime and nighttime continence were achieved in 13/13 
$(100 \%)$ and 12/13 (92\%), respectively. Chronic urinary retention was not noted. The urodynamic parameters were comparable in the available patients with and without genital preservation. Sexual function was detected as significantly better in these patients than in others without genital preservation. ${ }^{9}$ In their series, one of the patients underwent RARC. In a review, genitalia sparing $\mathrm{RC}$ was found to have additional functional benefits in carefully selected patients. ${ }^{10}$

In a large open series, Jentzmik et al reported the results of 241 women with organ-confined bladder cancer underwent radical cystectomy with subsequent urinary diversion including $50(20.7 \%)$ with ureterocutaneostomy, $70(29.0 \%)$ with ileal conduit, and 121 (50.2\%) with ileal neobladder recon-struction. Daytime and nighttime urinary continence were reported by 82.4 and $76.5 \%$, respectively, after a median follow-up of 6.1 years. These rates were higher than our series. In our series, the median follow-up time was shorter. With longer follow-up times, higher continence rates are expected. ${ }^{11 .}$

Currently, the number of centers performing genital sparing RARC is very limited. In 2004, Menon et al described RARC with preservation of urethra, uterus, vagina, and both ovaries in 2 female patients. Urinary reconstruction was performed extracorporeally. ${ }^{2}$ Hosseini et al stated that in cases when a vaginal-sparing dissection is planned and no suspicion of tumor invasion towards the uterus exists, the uterus can be dissected separately. ${ }^{1}$ Tuderti et al performed 11 genital sparing RARC. Daytime and nighttime urinary incontinence were $90.9 \%$ and $86.4 \%$, respectively. In the first year follow-up, $72.7 \%$ of the females were sexually active. During a median 28 months of follow-up, no recurrences were detected. ${ }^{5}$ Hussein et al described a detailed step-by- step approach of another robot-assisted intracorporeal orthotopic, ileal neobladder which that followed the general principles of a "W"-configuration (Hautmann's) orthotopic neobladder. Among 5 patients, they reported no pT3/4 patients or surgical margin positivity. ${ }^{12}$ In our experience, operation times were acceptable in all cases. Surgical margin was positive in two patients with pT4a and pT4b diseases and sufficient LN yields were obtained in all cases. We had only few minor early postoperative complications. One of the patients who experienced post-renal failure between postoperative 31-90 days was treated with bilateral double-J stent insertion. Regarding functional outcomes, although all patients were sexually not active, they had promising daytime and night-time urinary continence. Although our surgical experience currently includes only five patients, we have shown that genitalia sparing RARC with intracorporeal Studer pouch reconstruction is a safe and feasible minimally invasive procedure with acceptable shortterm oncologic and functional outcomes.

\section{Conclusion}

An increasing number of publications exist in the literature related to robot-assisted laparoscopic radical cystectomy (RARC) in females, and, to date, very few authors have described gynecologic-tract sparing RARC. Although our surgical experience currently includes only five patients, we have shown that genitalia sparing RARC with intracorporeal Studer pouch reconstruction is a safe and feasible minimally invasive procedure with acceptable short-term oncologic and functional outcomes in carefully selected patients.

\section{Funding}

There is no funding to report.

\section{Disclosure}

The authors reported no conflicts of interest for this work.

\section{References}

1. Hosseini A, Adding C, Nilsson A, Jonsson MN, Wiklund NP. Robotic cystectomy: surgical technique. BJU Int. 2011;108(6 Pt 2):962-968. doi:10.1111/j.1464-410X.2011.10566.x

2. Menon M, Hemal AK, Tewari A, et al. Robot-assisted radical cystectomy and urinary diversion in female patients: technique with preservation of the uterus and vagina. J Am Coll Surg. 2004;198(3):386-393. doi:10.1016/j.jamcollsurg.2003.11.010

3. Roshdy S, Senbel A, Khater A, et al. Genital sparing cystectomy for female bladder cancer and its functional outcome; a seven years' experience with 24 cases. Indian J Surg Oncol. 2016;7(3):307-311. doi:10.1007/s13193-015-0462-4

4. Kulkarni JN, Rizvi SJ, Acharya UP, Kumar KS, Tiwari P. Gynecologic-tract sparing extra peritoneal retrograde radical cystectomy with neobladder. Int Braz J Urol. 2008;34(2):180-187; discussion 187-190. doi:10.1590/S1677-55382008000200008

5. Tuderti G, Mastroianni R, Flammia S, et al. Sex-sparing robot-assisted radical cystectomy with intracorporeal padua ileal neobladder in female: surgical technique, perioperative, oncologic and functional outcomes. J Clin Med. 2020;9(2):2. doi:10.3390/jem9020577

6. Salem H, El-Mazny A. A clinicopathologic study of gynecologic organ involvement at radical cystectomy for bladder cancer. Int $J$ Gynaecol Obstet. 2011;115(2):188-190. doi:10.1016/j.ijgo.2011.05.026

7. Ali-El-Dein B, Abdel-Latif M, Mosbah A, et al. Secondary malignant involvement of gynecologic organs in radical cystectomy specimens in women: is it mandatory to remove these organs routinely? $J$ Urol. 2004;172(3):885-887. doi:10.1097/01.ju.0000133986.29257.bf

8. Bai S, Yao Z, Zhu X, et al. The feasibility and safety of reproductive organ preserving radical cystectomy for elderly female patients with muscle-invasive bladder cancer: a retrospective propensity score-matched study. Urology. 2019;125:138-145. doi:10.1016/j.urology.2018.09.035

9. Ali-El-Dein B, Mosbah A, Osman Y, et al. Preservation of the internal genital organs during radical cystectomy in selected women with bladder cancer: a report on 15 cases with long term follow-up. Eur J Surg Oncol. 2013;39(4):358-364. doi:10.1016/j.ejso.2013.02.004 
10. Niver BE, Daneshmand S, Satkunasivam R. Female reproductive organ-sparing radical cystectomy: contemporary indications, techniques and outcomes. Curr Opin Urol. 2015;25(2):105-110. doi:10.1097/MOU.0000000000000146

11. Jentzmik F, Schrader AJ, de Petriconi R, et al. The ileal neobladder in female patients with bladder cancer: long-term clinical, functional, and oncological outcome. World J Urol. 2012;30(6):733-739. doi:10.1007/s00345-012-0837-x
12. Hussein AA, Ahmed YE, Kozlowski JD, et al. Robot-assisted approach to 'W'-configuration urinary diversion: a step-by-step technique. BJU Int. 2017;120(1):152-157. doi:10.1111/bju.13824

Robotic Surgery: Research and Reviews

\section{Publish your work in this journal}

Robotic Surgery: Research and Reviews is an international, peer reviewed, open access, online journal publishing original research, commentaries, reports, and reviews on the theory, use and application of robotics in surgical interventions. Articles on the use of supervisorycontrolled robotic systems, telesurgical devices, and shared-control systems are invited. The manuscript management system is completely online and includes a very quick and fair peer review system, which is all easy to use. Visit http://www.dovepress.com/testimonials. php to read real quotes from published authors.

Submit your manuscript here: https://www.dovepress.com/robotic-surgery-research-and-reviews-journal 